increases the appetite and the power of assimilation, it has no influence in increasing the richness of the blood in hremoglobin (Moriez). Lastly, quinine and strychnine, although not hrematinies in the strict sense of the word, are very valuable as adjuvants; and the same may be saicl of the mineral acids and bitters.

The st:bject of transfusion of blood is one which demands the most thorough examination in reference to the treatment of anxmia. In some forms of symptomatic anxmia, especially when the cause is a sucken loss of blood, there can be no question either as to the propriety of transfusion or its efficacy in saving life. It is another matter, however, when we have to deal with the insidious and progressive anamia, which has been denoted by the term "pernicious". Transfusion ins not yet been employed to a very large extent in such cases, and it has often failed of success. I find that it was performed in 20 out of 110 cases, to which I referred in my last lecture, and that the six recoveries (one of which was not permanent) all occurred in Quincke's practice.

I think his success in these cases may be attributed to his having re. surted to transfusion at a less advanced stage of the disease than is usually the case. Ilis first cases were invariably fatal, and in his com. mentary upon them he pointed out the inutility of deferring transfusion until the heart is alrcady advanced in degeneration, and restoration is wcil nigh hopeless. In one of his papers, where he summarises the resills of five such transfusions, he says that defibrinated human blood was u.ed, and from So to 100 cubic centimètres injected into the radial artery. (Professor Schäfer, in his valuable report to the Obstetrical Society of last year, advocates immediate injection from artery to artery as far preferable to intravenous injection, whether mediate or immediate. It will be seen that Quincke performs an operation the same as regards the recipient of the blood; but he uses defibrinated blood, and does not practice the immediate transference.) Usually, during the transfusion, the face becomes flushed, the pulse slow, and somctimes very weak, almost a state of collapse occurring. In three cases a rigor, followed by fever, occurred a short time after the operation. On two occasions the transfusion was followed by hæmatinuria and casts in the urine, but not by the escape of actual blood-corpuscles. In one of these cases the amount of hxmoglobin passing off by the urine was estimated at one-sixteenth of that injected; and Quincke poin:s out that similar effects follow, according to Ponfick, the transfusivi of an animal with blood from another of different spccics. In sone cases petechial and other hxemorrhages follow transfusion. Quincke seems to be firmly of the opinion that by transfusion the blood is rcinforced with functionally active elements, and the results of his cases apparently bear out that opinion. Transfusion, then, is a correct and rational procedure, and one which we are bound to adopt in these cases of progressive idiopathic anamia, if no ordinary treatment makes any impression on them. But its rcsults can never be expected to be crowned with such success as in cases of acute anxmia following hxemorrhage, for the simple reason that we are dealing with a disease the origin of which lies deep in the organism, and the effects of which are already manifest in grave degenerative changes in the heart. Perhaps the less heroic measure of administering enemata of defibrinated ox blood, which was advocated by the Therapeutical Society of New York ss of great valuc in conditions of exhaustion and anxmia, may be found of service. $\Lambda$ t any rate, it deserves the careful trial which Dr. Sanson in this country has claimed for it.

It only remains for me to thank you for the kind attention you have given me during this imperfect attempt to illustrate the leading features of anemia. I have consciously left many points untouched, whilst many of which I have spoken are capable of much fuller analysis than I have attempted. For all the shortcomings of what has been ittle more than an abstract of the present state of knowledge on this subject, I ask your indulgence.

Vaseline and Artificial Eyes.-Dr. Chas. S. Turnbull recommends pure vaseline to all wearers and fitters of artificial eyes. It is infinitcly superior to pure water, oils, or unguents, as a lubricant, not only for daily use, but also for facilitating the tedious and irritating process of fitting and matching. It lessens wear and tear. It allay's the vurning sensation often experienced toward night. It is of particuliar value in preventing the drying of mucus upon the surface of the eye, an occurrence most annoying to wearcrs, as well as to their surgeons; and last, but not least, it serves as a convenient and desirable vehicle for the application of anodyne or astringent remedies. It should be applied once or twice daily, according to the employment of the wearer ; and by mechanics, mill hands, persons working out of doors, or in disty or dry atnocspheres, has been found of more than ordinary valuie.

\section{ON PARALYTIC CHOREA.*}

BY W. R. GOWERS, M.D., F.R.C.P., Assistant Physician to University College Hospital; Physician to the National Hospital for the Paralysed and Epileptic.

MY object in this paper is to direct attention to a variety of chorea which sometimes presents a difficulty in diagnosis.

In an ordinary well-marked case of common chorea, we may usually recognise three symptoms: spontaneous movement, inco-ordination of voluntary movement, and muscular weakness. These three symptoms are not always proportioned in degree; any one of them may so predominate as to give a special aspect to the case. The form which I am about to describe is one in which the last element-muscular weakness-predominates, and in which the other elements-muscular spasm and inco-ordination-are so slight, as to be apparently absent; so that the muscular weakness appears to be the only symptom.

I will first describe briefly the characters of a few examples of this form of affection.

CASE 1. - John F., aged 14, applied for treatment in July 1879, because he had "lost the use of his left hand". The boy presented the characteristic signs of inherited syphilis : scarred mouth, one notched incisor, cicatrices of old ulceration on the uvula, iritic adhesions in the left eye, and evidence of previous choroiditis in both eyes. The left arm hung by his side. He could move it by an effort, but it was con. siderably weaker than the right. and this also was weaker than normal. The weakness was said to have come on gradually about a fortnight previously. There was no paralysis of face or leg. On first examina. tion, no twitching or choreic movement could be observed. Voluntary movements were perfectly steady. On subsequent close watching, however, an occasional slight movement of the thumb, or of pronation was observable in the right hand, but none in the left. When he held both arms straight forward for a time, a slight occasional twitch was noted in each hand. He was given iodide of potassium and strychnia. A week later, the left arm was stronger, and could be used better. It did not prevent a greater degree of twitching. The spontaneous movements in the right arm had increased considerably, and now presented a distinct chorea-like character. He did not, however, present himself again.

CASE II.-Tane P., a girl aged 13 , was brought on June 9th, 1879, having, it was said, "lost the use of her left arm" for three months; the onset of the weakness had been gradual. The arm was considerably weaker than the other, the grasp being less than half the strength of the right. Close examination did not reveal the slightest spontaneous movement. It was, however, ascertained that, earlier in the affection, she would occasionally drop things out of the hand, although no history of distinct choreic movements could be elicited. She was said to have had rheumatic fever at four years of age. The heart was healthy. She gradually, but slowly, regained considerable power of using the arm; but with the power, slight spontaneous movements appeared, and then gradually subsided. The symptoms had only disappeared four months after the commencement of treatment, so it may be doubted whether this exercised much influence. First strychnia, and afterwards arsenic, were given.

CASE III,-Emma D., aged 9, was brought on May 1879, on account of weakness of the left arm, which hung useless by her side. She never attempted to use it, although by an effort she could grasp with slight force. The weakness had come on gradually, four months previously. Not the least spontaneous movement could be observed in it. Co-ordination of movement was perfect. Nevertheless, when she grasped the hand, it could be felt that the movement of the fingers was not quite equal. In the legs, especially the left, there was slight twitching. Inquiry elicited that, during the first few weeks of the affection, there had been more spontaneous movement, and that this had lessened as the weakness came on. There was a loud mitral murmur. Strychnia was given, and in a month she was practically well, although the power of the arm was not quite so good as that of the other.

CASE IV.--Ellen B., aged 14, brought on March 15th, 1880, on account of "loss of the use of her left arm", of a few weeks' duration, and gradual onset. The arm hung by her side, and she made no attempt to use it. She could by an effort raise it, but with very little power; and the hand was so feeble, that no sensible pressure could be exerted in the grasp. The leg was strong. The face presented no weakness; the tongue no deviation. At first, no movements

* A paper read before the Section of Medicine at the meeting of the British Mcdical Association at Cambridge, August i 330 . 
were observed in the arm, but, on watching closely for a little time, an occasional slight twitch was observed. Occasional sighing was also noted. There was no cardiac murmur. She improved very little as an out-patient during two months' treatment, and there gradually developed distinct choreic movements in the right arm, without much weakness. She was then admitied into the county branch of the Hospital, and rapidly improved; the movements in the right arm ceased, and the left arm became much stronger, without preventing distinct choreiform spasm; the power of grasp increasing in three weeks from 0 to 17 kilogrammes.

CASE v.-L. W., aged 7, was brought to the Queen Square Hospital in February 1879, with the statement that he had, during the previous two months, gradually lost the use of the right hand. The hand was found to be distinctly weaker than the left. There was no inco-ordination of movement; and, at first sight, no choreiform movements. Close observation, especially when he was holding an object with the right hand, detected a slight occasional twitch. Strychnia was given, and he gradually improved; power increasing, and the spontaneous movements ceasing to be obscrved. In two months' time, he was well.

These cases speak for themselves, and it is hardly ncccssary for me to point out their chief characteristics. The age at which the affection may occur is that at which chorea is the most common, seven to fifteen years. As a rule, gradual loss of the use of one arm alone attracts the attention of the friends. In some cases, the sudden dropping of objects, 50 characteristic of the imperfect muscular control of chorea, is noted early. In rare cases, slight clonic spasm occurs in the affected arm at first, and passes off subsequently. The loss of power may be very great and real, or it may be much less than the loss of use of the limb would suggest. In these cases, there is rather inaction than paralysis. The affection is usually confined to one arm; there is no hemiplegic weakness; no paralysis of face, tongue, and leg. Both arms may be affected, but one is always weaker than the other. Close and continued observation will generally detect an occasional slight choreic twitch, even in the arm which is weak. but often more conspicuous in the other arm, which is the less weak, or even strong. Sometimes, slight clonic contractions may be noted in the legs. The affection may pass off without choreiform movements being more conspicuous. Frequently, however, these subsequently came on; and they may cren be more marked as the power in the arm becomes greater.

Whenever a child of this age suffers from gradual loss of power in the arm, and presents no weakness in face, tongue, or leg, the disease, as far as I have seen, is always chorea. If the nature of the case be suspected, confirmatory evidence-slight occasional spasm-if looked for, will commonly be at some time detected, either in the weak arm or in the other.

The course of these cases is often tedious; and, as the choreoid movements may increase as the weakness passes off, the patients often seem, to the friends, to be getting worse when really getting better. I have never seen this form pass on into severe general chorea.

\section{ON FRACTURE OF THE NECK OF THE HUMERUS, AS A COMPLICATION OF DISLOCATION OF} THE SHOULDER.*

BY EDWARD H. BENNETT, M.D., Professor of Surgery in the University of Dublin; Surgeon to Sir Patrick Dun's

THE examples of fracture of the neck of the humerus, occurring as a complication of dislocation of the head of the bone, which I present to the meeting, are of interest for their rarity, and, more particularly, for the light which they throw on the mode of production of this accident

The difficulties of diagnosis and of treatment, which attend this complicated injury, render it desirable that every opportunity should be availed of that may extend our knowledge of its pathology; Ineed not, therefore, offer any apology for occupying the attention of the meeting for a few minutes, while I point out the leading features of five examples which our Dublin collections contain. The first question which arises in the study of the injury has reference to the order of the occurrence of the fracture and dislocation. Which takes place firstthe fracture or the dislocation? Sir Astley Cooper answers, withou hesitation : "This accident generally occurs in the following way. A person falls and pitches with violence on his shoulder, or a heavily laden carriage passes over it. By the first impression of the accident the os humeri is dislocated; and, by a second, the neck of the bone is broken, and the head detached and lodged in the axilla." Sir Astley

\footnotetext{
- Read in the Section of Surgery at the Annual Meeting of the British Medical
} Association in Cambridge, August 1880. does not assign any reason for this assertion; nor do I know that any case is recorded in which the second cause which he describes has leen observed: the passage of a heavily-laden carriage over the shoulder. On the contrary, Malgaigne states distinctly that the cause of the accident has in every case been a fall, probably on the shoulder. Delpech, who first recorded with accuracy a case of this kind, proved, by dissection, not only the possibility of the fracture of the anatomical neck of the humerus, but also the possibility of dislocation of the humerns backwards-facts resting, in his day, on doubtful evidence. He discusses the question of the order of occurrence of the two lesions; and expresses the opinion that the first step is the production of the fracture - the second, the dislocation. "The fracturing force itself, and some chance movements of the arm, are sufficient to produce the dislocation." It is curious to observe that the opinions of Delpech and Cooper, so directly opposite, have been based on the cxamination of instances of the fracture in which the dislocations took place in opposite directions : in Delpech's, backwards; in Cooper's, forwards.

The explanation offered by Delpech is, that the bone, already rotated inwards by some accidental circumstance of the fall, strikes against the ground-so that the outcr margin of its head bears the shock, while the inner rests against the contour of the glenoid carity; that so the fracture of the anatomical neck is determined, and is followed by the protrusion of the head of the bone through the capsule. Such explanation might readily apply to cases in which the head of the brne is dislocated backwards; but, if we consider the more numerous cases of fracture with dislocation forwards, this explanation fails; for, in these cases, the head of the bone cannot be compresserl between the ground and the glenoid cavity. If such were the mode of production, we should expect, in some recorded case, to find comminutions of unc or both fragments present; but I find no mention of this condition; it is absent from the specimens before us. Malgaigne avoids this question in his detailed account of the injury; in his chapter on luxations, complicated by fractures in general, he disposes of it thus: "It is very difficult to define exactly the mechanism by which the two lesions, dislocation and fracture, take place; to determine whether the two lesions are simultaneous, or which precedes the other; whether they are dac in direct or to indirect injury."

Dr. R. W. Smith, in treating of "fractures of the humcrus in the vicinity of the shoulder-joint", onits all mention of this fracture. He describes at length one of the specimens which I now exhibit, anid concludes that it is an impacted intracapsular fracture-a conclusion which, I think, a comparison of the specimen with the others on the table, will demonstrate to be incorrect.

The study of the recorded cases, in which dissection has verified the injury with the detailed examination of thesc spccimens, leads me to conclude that the combination of injuries is due to a constant cause, and that this cause acts after the dislocation has occurred. My first specimen suggests this cause, and also its time of action. In this, a recent intracoracoid dislocation, in which the head of the bone has been left undisturbed in its dislocated position, there exists a partial fracture of the humerus in the part of the anatomical neck which is applied against the border of the glenoid cavity of the scapula, It will be seen, at a glance, that the fracture has been produced by pressure of the bone against the edge of the glenoid cavity; and that, from its extent and position, the fracture must have occurred after the bone had been dislocated. This specimen I obtained from the body of a man who fell twenty feet into the empty hold of a vessel, lighting on the kelson. As he tripped and fell, it was noticed by his fellow-workmen that he had the hand of the injured side in his breeches-pocket. He died of injuries to the head within three days, the dislocation remaining unreduced. It will be noticed that the coracoid process has also been broken by the pressure of the humerus against its tip. In the disscction, no superficial injuries of the coverings of the shoulder were visible, only the deeper lacerations, and ecchymoses due to the dislocation. It is interesting to note here that, in Delpech's case, it was ascertained that the injury was caused in a limb which was maintained in a constrained position fixed to the side. In my case, a more powerfully acting force might have extended the fracture, so as to detach the head, with portion of the bone beneath it-leaving, however, the great tubcrosity intact. My next two specimens present fractures of this kind. In both, the head of the humerns is in the position of intracoracoid dislocation; while, in each, the path of fracture starts from the anatomical neck, between the great tuberosity and head, and so passes obliquely through the upper extremity of the bone; instead of following the anatomical neck exactly, the fractures pass through the bicipital groove, so having the lesser tuberosity attached to the head. In one, but a very little piece of the shaft has been detached with the dislocated head from its posterior and in. ternal side; in the other, a longer splinter, just two inches in length. In the first, the long tendon of the biceps muscle was buried in the 